\title{
Nonlocal effect and dimensions of Cooper pairs measured by low-energy muons and polarized neutrons in type-I superconductors
}

\author{
V. Kozhevnikov, ${ }^{1, *}$ A. Suter, ${ }^{2}$ H. Fritzsche, ${ }^{3}$ V. Gladilin, ${ }^{4}$ A. Volodin, ${ }^{5}$ T. Moorkens,${ }^{5}$ M. Trekels, ${ }^{6}$ J. Cuppens, ${ }^{5}$ B. M. Wojek,,${ }^{2, \dagger}$ \\ T. Prokscha, ${ }^{2}$ E. Morenzoni, ${ }^{2}$ G. J. Nieuwenhuys, ${ }^{2,7}$ M. J. Van Bael, ${ }^{5}$ K. Temst,${ }^{6}$ C. Van Haesendonck, ${ }^{5}$ and J. O. Indekeu ${ }^{8}$ \\ ${ }^{1}$ Tulsa Community College, Tulsa, Oklahoma 74119, USA \\ ${ }^{2}$ Paul Scherrer Institute, CH-5232 Villigen PSI, Switzerland \\ ${ }^{3}$ Canadian Neutron Beam Centre, National Research Council Canada, Chalk River Laboratories, Ontario, Canada KOJ1JO \\ ${ }^{4} T Q C$, Universiteit Antwerpen, BE-2610 Antwerpen, Belgium \\ ${ }^{5}$ Laboratory of Solid-State Physics and Magnetism, KU Leuven, BE-3001 Leuven, Belgium \\ ${ }^{6}$ Institute for Nuclear and Radiation Physics, KU Leuven, BE-3001 Leuven, Belgium \\ ${ }^{7}$ Kamerlingh Onnes Laboratory, Leiden University, 2300 RA Leiden, The Netherlands \\ ${ }^{8}$ Institute for Theoretical Physics, KU Leuven, BE-3001 Leuven, Belgium
}

(Received 16 September 2012; revised manuscript received 20 February 2013; published 11 March 2013; corrected 26 March 2013)

\begin{abstract}
The Pippard coherence length $\xi_{0}$ (the size of a Cooper pair) in two extreme type-I superconductors (In and $\mathrm{Sn}$ ) was determined directly through high-resolution measurement of the nonlocal electrodynamic effect combining low-energy muon spin rotation spectroscopy and polarized neutron reflectometry. The renormalization factor $Z=m_{c p}^{*} / 2 m$ ( $m_{c p}^{*}$ and $m$ are the mass of the Cooper pair and the electron, respectively) resulting from the electron-phonon interaction, and the temperature-dependent London penetration depth $\lambda_{\mathrm{L}}(T)$ were determined as well. An expression linking $\xi_{0}, Z$, and $\lambda_{\mathrm{L}}(0)$ is introduced and experimentally verified. This expression allows one to determine experimentally the Pippard coherence length in any superconductor, independent of whether the superconductor is local or nonlocal, conventional or unconventional.
\end{abstract}

DOI: 10.1103/PhysRevB.87.104508

PACS number(s): 74.25.Ha, 74.78.-w, 78.70.-g

\section{INTRODUCTION}

A core concept of superconductivity is Cooper pairing of electrons. Cooper pairs have a characteristic size $\xi_{0}$, the Pippard coherence length, and an effective mass $m_{c p}^{*}=2 Z m$, where $m$, being close to the free-electron mass $m_{e}$, is the Coulomb and band-structure effective mass of the electrons and $Z$ is the renormalization factor, a measure of the electronboson coupling strength. ${ }^{1}$ Only weakly dependent on temperature $T^{2,3}, \xi_{0}$ provides a reference length for the fundamental length scales, including the London penetration depth $\lambda_{\mathrm{L}}(T)$, characterizing the decay of a penetrating magnetic field and associated with number density of Cooper pairs $n_{c p}$. In units of $\xi_{0}, \lambda_{\mathrm{L}}(T)$ determines whether the superconductor is described by local or nonlocal electrodynamics, and $\lambda_{\mathrm{L}}(0)$ determines whether it is type I or type II. ${ }^{2}$ To ensure consistency $\xi_{0}, Z$ and $\lambda_{\mathrm{L}}(T)$ should be measured simultaneously. As of today these parameters have not been simultaneously and directly measured in any superconductor.

A phenomenon stemming immediately from the finite size of the Cooper pairs and therefore providing a principal possibility for the direct determination of $\xi_{0}$ is an effect of nonlocal electrodynamics in the profile of the magnetic field $B(z)$ ( $z$ is the distance from the sample surface) penetrating into the superconductor in the Meissner state. This "nonlocal effect," first predicted by Pippard ${ }^{4}$ and following from the Bardeen-Cooper-Schrieffer (BCS) theory, manifests itself in the deviation of $B(z)$ from the exponential decay applicable in the local or London limit. ${ }^{2}$ Moreover, $B(z)$ in nonlocal superconductors is predicted to change sign at a certain depth. Most superconductors are local and for those $\xi_{0}$ cannot be measured, although Kosztin and Leggett have shown that under certain conditions the nonlocal effect may also take place in unconventional superconductors with nodes in the energy gap. ${ }^{5}$
When a superconductor is in the Meissner state, an external magnetic field $B_{0}$ is completely screened within the sample interior due to a persistent current running in a thin surface layer over which the field decays to zero. The layer thickness is of the order of the "magnetic penetration depth" $\lambda \equiv$ $B_{0}^{-1} \int_{0}^{\infty} B(z) d z$. If the size of the Cooper pairs is small $\left(\xi_{0} \ll \lambda\right)$, the relationship between the current density and the vector potential can be treated as local. Then the field decays as $\exp \left(-z / \lambda_{\mathrm{L}}\right)$, where $\lambda_{\mathrm{L}}=\sqrt{\Lambda c^{2} / 4 \pi}$ is the London penetration depth ( $c$ is the speed of light). In the London theory the phenomenological parameter $\Lambda$ is a function of the mass $m_{s c}$ and the number density $n_{s c}$ of superconducting electrons, none of the two being well defined. ${ }^{2}$ We want to remind the reader that $m_{s c}$ is not $m_{c p} / 2$ and $n_{s c}$ is not $2 n_{c p}$. The number density $n_{s c}$ is an "effective number of electrons," which can be determined from the value of $\lambda_{\mathrm{L}}^{2}\left(=m_{s c} c^{2} / 4 \pi n_{s c} e^{2}, e\right.$ being the electron charge) assuming that $m_{s c}=m_{e}{ }^{6}$ However, due to dimensional correctness, the London's formula correctly describes the dependence of the penetration depth on the mass and density of the Cooper pairs $\left(\lambda_{\mathrm{L}}^{2} \sim m_{c p} / n_{c p}\right)$.

The local approximation is applicable to superconductors with Ginzburg-Landau parameter $\kappa \gtrsim 1 .{ }^{7}$ For superconductors with smaller $\kappa$ the Cooper pair size is no longer negligible and the current density is determined by the vector potential averaged over a region of dimension $\xi_{0}$. The nonlocality leads to deeper field penetration and to distortion of the $B(z)$ shape as was mentioned above. The nonlocal effect is approximately proportional to $\kappa^{-1 / 3}$ and for majority superconductors is very small ${ }^{2}$ it is most pronounced in extreme type-I or Pippard superconductors, such as $\mathrm{Al}(\kappa \approx 0.01)$, In $(0.07)$, and $\mathrm{Sn}$ (0.11). The field profiles for $\mathrm{Al}$ following from local and nonlocal theories are shown in Fig. 1 (see also Ref. 3). In the pure limit (elastic mean free path $\ell \gg \xi_{0}$ ) nonlocal 


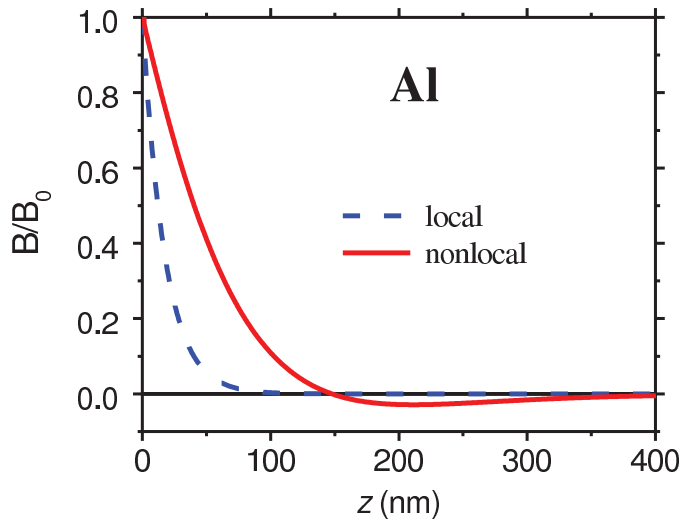

FIG. 1. (Color online) Magnetic field profiles for aluminium at $T=0$ calculated from London (local) and BCS (nonlocal) theories with $\lambda_{\mathrm{L}}$ and $\xi_{0}$ taken from Ref. 8.

superconductors $B(z)$ is a function of $\lambda_{\mathrm{L}}(T)$ and $\xi_{0},{ }^{2}$ each of which depends on mass of Cooper pairs. Therefore, knowledge of $B(z)$ in such materials at different temperatures allows one to determine $\lambda_{\mathrm{L}}(T)$ and $\xi_{0}$ and to find $Z$ as it is shown in the next section. Determination of the key microscopic parameters $\lambda_{\mathrm{L}}(T), \xi_{0}$, and $Z$ through measurement of $B(z)$ in two Pippard superconductors In and Sn is the goal of this work. Two superconductors are needed for mutual verification of experimental results. In and Sn are chosen due to their convenience for experiments performed in this work and because they are the strongest nonlocal superconductors after Al.

An observation of the sign reversal in $B(z)$ via measurements of an ac magnetic field leaking through a $1.8 \mu \mathrm{m}$ thick tin film was reported in Ref. 9. However, the validity of this observation is questionable. ${ }^{10}$ In a subsequent paper ${ }^{11}$ the authors, as a matter of fact, discard this result mentioning that they failed to reproduce it with other 24 identically fabricated samples and by not providing new data points for the "good" sample.

A first determination of the penetration-layer characteristics in a nonlocal superconductor has been achieved by Doezema et al. ${ }^{12,13} B(z)$ in a single-crystal Al sample was studied utilizing a resonance magnetoabsorption technique. In this technique a fixed-frequency microwave absorption is measured versus an external magnetic field applied parallel to the sample surface. Due to the resonant character, the technique is very sensitive. However, for the same reason, only a few points of an effective potential $V_{e f f}(z)$ [linked with $B(z)$ calculated from the BCS theory] trapping quasiparticles in the penetration layer were identified. Besides, the interpretation of the experimental spectra requires detailed knowledge of parameters of the normal state, whose accuracy is difficult to quantify. This work of Doezema et al. is an undisputed experimental masterpiece; its results are consistent with the nonlocal theory, although obtained quantitative parameters remain to be verified via independent measurements.

The appearance of polarized neutron reflectometry (PNR) ${ }^{14,15}$ and low-energy muon spin rotation spectroscopy $(\mathrm{LE}-\mu \mathrm{SR})^{16,17}$ techniques qualitatively changed the landscape of studies of surface magnetization. In particular, these techniques enable direct measurements of $B(z)$. The PNR and LE- $\mu$ SR techniques are based on different principles, hence complementing each other and providing a possibility for mutual cross checking of experimental results.

The nonlocal effect was unambiguously confirmed for the first time by LE- $\mu$ SR measurements on $\mathrm{Pb}, \mathrm{Ta}$, and $\mathrm{Nb},{ }^{3,18}$ and soon after by PNR measurements in In. ${ }^{10}$ In Refs. 3,18 a first attempt was made to infer $\xi_{0}$ from $B(z)$ in $\mathrm{Pb}$. However, due to an unresolved issue of systematic errors only statistical errors were estimated. One can overcome this problem by combining LE- $\mu$ SR and PNR measurements since in the latter the systematic errors can be excluded. Therefore the method of our choice is to combine LE- $\mu$ SR and PNR measurements of $B(z)$; such an approach enables an independent verification of the inferred values of $\xi_{0}$ and $\lambda_{\mathrm{L}}$ and an estimate of their total uncertainties.

The paper is organized as follows: Section II presents an approach to deduce the renormalization factor $Z$; a brief description of the LE- $\mu$ SR and PNR techniques and approaches for the treatment of experimental data are presented in Sec. III; the sample characteristics are described in Sec. IV; the experimental results are presented and discussed in Sec. V; Sec. VI contains a summary and outlook.

\section{RENORMALIZATION FACTOR}

Electrons near the Fermi surface are dressed by a cloud of virtual phonons, leading to an enhancement of their effective mass and consequently to a reduction of the Fermi velocity $v_{\mathrm{F}}$. Below the critical temperature $T_{c}$ the phonon-mediated attraction of electrons exceeding their screened Coulomb repulsion results in the formation of Cooper pairs. In BCS theory the electron-electron coupling is weak: $N(0) V \ll 1$, where $V$ is the pairing potential and $N(0)$ is the single-spin electron density of states at the Fermi surface, which can be obtained from specific-heat measurements. Eliashberg's strong-coupling theory (SCT) is free from this limitation and agrees better with experimental results. ${ }^{1}$

In SCT the effective mass of electrons near the Fermi surface is $m^{*}=Z m$ and consequently the effective mass of the Cooper pairs is $m_{c p}^{*}=2 \mathrm{Zm}$. Correspondingly (as can be seen from the London's formula for $\lambda_{\mathrm{L}}$ and the Pippard/BCS formulas for $\xi_{0}^{2}$ ), $\lambda_{\mathrm{L}}$ and $\xi_{0}$ are renormalized with respect to their values in the weak-coupling $(w c)$ limit as follows,

$$
\begin{aligned}
\lambda_{\mathrm{L}} & =\sqrt{Z} \lambda_{\mathrm{L}}^{w c}, \\
\xi_{0} & =\xi_{0}^{w c} / Z .
\end{aligned}
$$

If $\lambda_{\mathrm{L}}(T \rightarrow 0)$ is measured, the factor $Z$ can be inferred from Eq. (1). Neglecting effects of anisotropy, $\lambda_{\mathrm{L}}^{w c}(0)$ can be obtained using $\Lambda$ for electrons not colliding with the lattice. ${ }^{19,20}$ This leads to

$$
\lambda_{\mathrm{L}}^{w c}(0)=\sqrt{\frac{3 c^{2}}{8 \pi e^{2} N(0) v_{F}^{2}}} .
$$

On the other hand, if $\xi_{0}$ is measured, $Z$ can be calculated from Eq. (2) using the BCS definition $\xi_{0}^{w c} \equiv \hbar v_{\mathrm{F}} / \pi \Delta(0)=$ $0.18 \hbar v_{\mathrm{F}} / k_{B} T_{c}$, where $\Delta(0) \equiv \Delta(T=0)$ is the energy gap and $h=\hbar \times(2 \pi)$ and $k_{B}$ are the Planck and Boltzmann constants, respectively. 

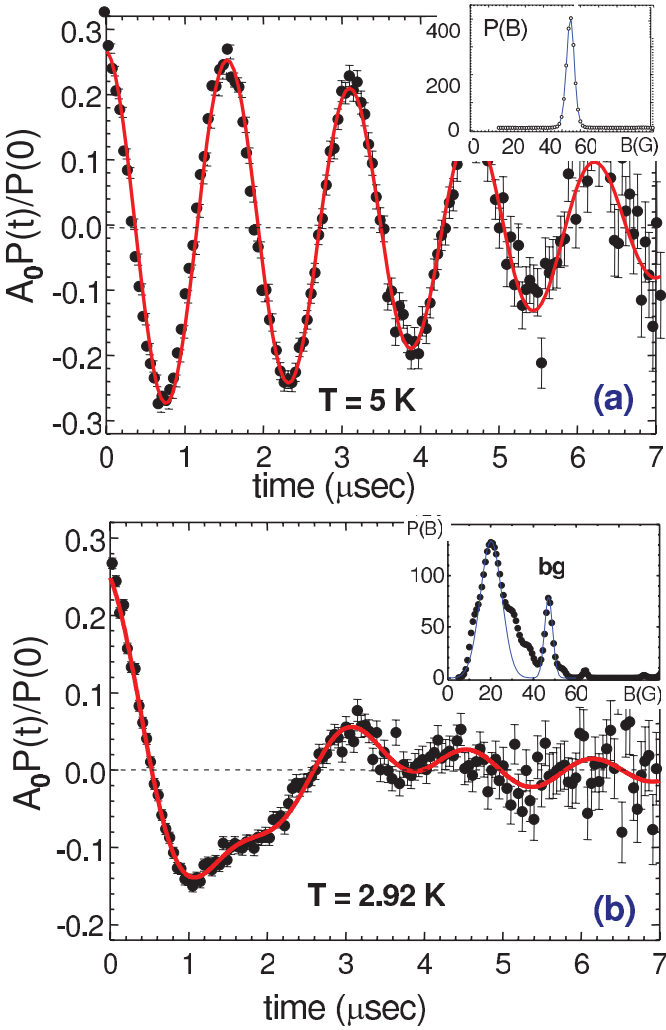

FIG. 2. (Color online) Time spectra of muons with energy of $22 \mathrm{keV}$ at an applied field of $47 \mathrm{Oe}$ in the normal state (a) and in the Meissner state (b) measured on the IN-1 sample. The curves are fits to the Gaussian (a) and the time-domain (b) models. The insets present the field spectra $P(B)$. In the inset in (b) $b g$ is the background contribution, while the curve represents two Gaussian peaks to approximate the experimental spectrum. $A_{0}$ is the maximum observable asymmetry of the muon decay.

Unfortunately neither of these approaches is applicable for quantitative analysis since reliable calculation of $v_{\mathrm{F}}$ (the Fermi velocity averaged over the free area of the Fermi surface $S_{\mathrm{F}}$ ) is hardly possible due to the complex topology of $S_{\mathrm{F}}$ in polyvalent metals. ${ }^{8,21}$ However, if both $\lambda_{\mathrm{L}}(0)$ and $\xi_{0}$ are known, one can eliminate $v_{\mathrm{F}}$, thus obtaining

$$
Z=\frac{c^{2} \hbar^{2}}{12.5 \pi T_{c}^{2} e^{2} \gamma} \frac{1}{\lambda_{\mathrm{L}}(0)^{2} \xi_{0}^{2}},
$$

where it is taken into account that $N(0)=3 \gamma / 2 \pi^{2} k_{B}^{2}, \gamma$ being the Sommerfeld constant (the electron heat capacity coefficient). ${ }^{21}$ Hence Eq. (4) allows one to determine $Z$ from $B(z)$ in nonlocal materials. We want to stress that Eq. (4) is independent of the relationship between the current density and the vector potential and of the specific nature of the electron-electron pairing. Therefore Eq. (4) can be applied to any pure superconductor. For impure superconductors the influence of the finite mean free path can be accounted for via an effective range of coherence $\xi^{\prime}(\ell)=\left[\left(\alpha / \xi_{0}\right)+(1 / \ell)\right]^{-1}$, where $\alpha$ is a factor on the order of unity. 2,4

In SCT $Z=1+\lambda_{m}$, where $\lambda_{m}$ is a mass-enhancement parameter tabulated in Ref. 1 on the basis of electron tunneling experimental data. $\lambda_{m}$ can also be obtained from McMillan's equation for $T_{c}{ }^{8}$, but this approach is less reliable. ${ }^{1}$ Therefore, for nonlocal superconductors Eq. (4) builds a bridge between the $B(z)$ and the tunneling data, hence providing an independent test of the values of $\lambda_{\mathrm{L}}$ and $\xi_{0}$ inferred from the $B(z)$ data. On the other hand, the vast majority of superconductors are local. For those Eq. (4) allows one to determine $\xi_{0}$ from the measured values of $\lambda_{\mathrm{L}}(0)$ and $Z$ using, e.g., LE- $\mu$ SR and tunneling experiments, respectively.

\section{EXPERIMENTAL TECHNIQUES}

\section{A. $\mu$ SR and LE- $\mu$ SR}

$\mu$ SR makes use of polarized positive muons, $\mu^{+}$. Implanted into the host material they thermalize on a picosecond time scale without loss of the spin polarization. Similar to NMR, the $\mu^{+}$is acting as a local magnetic microprobe in the sample under investigation. The $\mu^{+}$is a radioactive particle with a lifetime of $2.2 \mu \mathrm{s}$, decaying into 3 particles ( 2 neutrinos and a positron). Due to the parity violation of the weak decay, the positron is preferentially emitted along the spin direction of the $\mu^{+} .{ }^{22}$ Hence, by time-ensemble averaging of $10^{6}-10^{7}$ muons, precession signals can be recorded which reflect the measured magnetic field at the muon site.

Surface muon based $\mu$ SR uses energetic $(\sim 4 \mathrm{MeV}) \mu^{+}$ with a stopping range in solids on the sub-mm scale, implying it is suitable for bulk studies. Low-energy $\mu$ SR (LE- $\mu$ SR) uses $100 \%$ polarized $\mu^{+}$of tunable energies in the $\mathrm{keV}$ range to study local magnetic properties in thin films. $\mathrm{keV}$ muons are obtained by a moderation technique. ${ }^{16,23}$ At these energies the implantation depth of the $\mu^{+}$ranges from a few $\mathrm{nm}$ to a few hundred $\mathrm{nm}$. The stopping range profile can be calculated by the Monte Carlo program TRIM.SP, which treats the $\mu^{+}$as a light proton. ${ }^{24,25}$

Due to the "short" lifetime of the muon, high-quality statistics of data sets can be collected in a rather short time ( $\lesssim 1$ hour). Another advanced feature of $\mu \mathrm{SR}$ is its ability to operate with small (few $\mathrm{mm}$ ) or mosaic samples. ${ }^{26,27}$ Complications in using this technique for $B(z)$ measurements

TABLE I. Parameters of the samples. $d$ is the thickness, $\sigma$ is the AFM measured rms roughness, $\varrho$ is the oxide layer thickness inferred from the neutron reflectivity measurements (see Ref. 10); RRR is the residual resistivity ratio; $\ell$ is the elastic mean free path.

\begin{tabular}{lccccccc}
\hline \hline sample & type & size $(\mathrm{cm})$ & $d(\mu \mathrm{m})$ & $\sigma(\mathrm{nm})$ & $\varrho(\mathrm{nm})$ & RRR & $\ell(\mu \mathrm{m})$ \\
\hline IN-1 & film & $2 \times 3$ & 2.5 & 5 & $\leqslant 1$ & 560 & 11 \\
IN-2 & film & $\varnothing 6$ & 3.3 & 6 & $\leqslant 1$ & 730 & 14 \\
SN & cryst & $1.5 \times 2$ & $2 \times 10^{3}$ & 2 & 7 & $>1300$ & $>12$ \\
\hline \hline
\end{tabular}



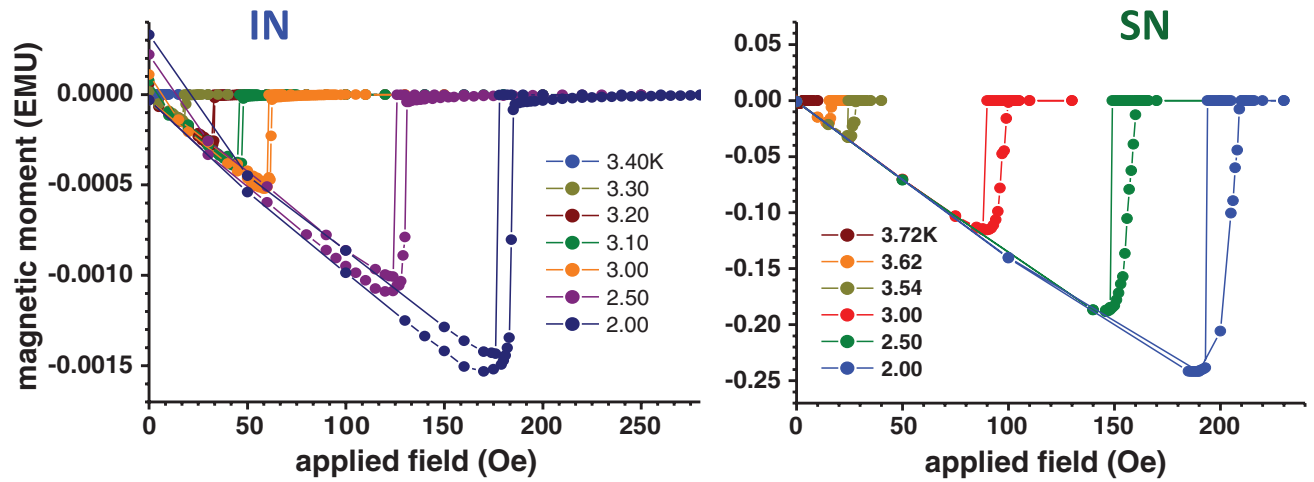

FIG. 3. (Color online) Magnetization of IN-1 and SN samples.

arise from the rather broad distribution of muon stopping distances, ${ }^{3}$ which makes it difficult to quantify uncertainties.

\section{B. LE- $\mu$ SR data treatment}

Typical time spectra of muon polarization $P$ in the normal and the Meissner states are presented in Fig. 2. We use two models to interpret these spectra: a Gaussian and a time-domain model.

In the Gaussian model the time dependence of the muon polarization $P(t)$ has the form

$$
\begin{aligned}
P(t)= & w \exp \left[-\frac{1}{2}\left(\sigma_{S C} t\right)^{2}\right] \cos \left(\gamma_{\mu} B_{S C} t\right) \\
& +(1-w) \exp \left[-\frac{1}{2}\left(\sigma_{B G} t\right)^{2}\right] \cos \left(\gamma_{\mu} B_{0} t\right),
\end{aligned}
$$

where $B_{S C}$ is the magnetic field at the muon site inside the sample, $B_{0}$ is the applied field, $\sigma_{S C}$ and $\sigma_{B G}$ are respective depolarization rates of the muons inside and outside (background) the sample, and $w$ is the statistical weight of muons stopped in the sample (in our experiments $w \geqslant 0.92$ ). The fitting parameters are $B_{S C}, \sigma_{S C}, \sigma_{B G}$, and $w$. The model assumes a Gaussian spectrum of the field; this is exact in the normal state [Fig. 2(a)]. In the Meissner state the model assumes different fields inside and outside the sample, both fields having a Gaussian spectrum. Although the real field distribution in the sample is not Gaussian, it still can be

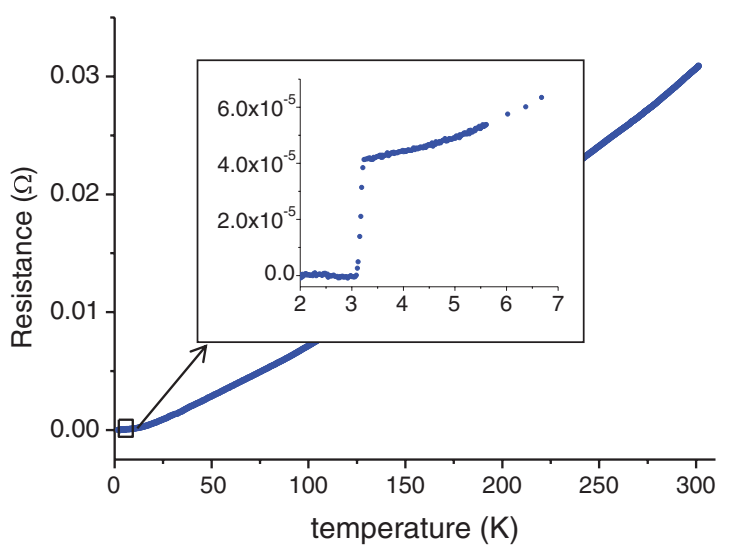

FIG. 4. (Color online) Temperature dependence of electrical resistance of IN-2 sample. approximated by a Gaussian peak [line in the inset of Fig. 2(b)], which makes the model sufficient for a qualitative analysis of the $B(E)$ data, where $E$ is the implantation energy of the muons. An additional uncertainty in using this model for a nonuniform $B(z)$ field arises from the distribution of stopping distances ${ }^{3}$ because there is no strict criterion to which depth $z$ the field $B$ extracted from the Gaussian fit should be assigned. It can be the mean value $\langle z\rangle$ of the implanted profile or its peak value $z_{p}$. As shown in Ref. 3 , the mean $\langle z\rangle$ is more appropriate for the analysis of field profiles in nonlocal superconductors.

The time-domain model assumes a priori a specific shape of the field profile following from theory. In the case of nonlocal superconductivity it is the Pippard/BCS magnetic field profile $B_{\mathrm{BCS}}(z) \equiv B_{\mathrm{BCS}}\left(z, \lambda_{\mathrm{L}}(T), \xi_{0}, \ell\right)$. The muon polarization $P(t)$ in the time-domain model has the form

$$
\begin{aligned}
P(t)= & w \exp \left[-\left(\sigma_{\mathrm{SC}} t\right)^{2} / 2\right] P_{\mathrm{BCS}}(t) \\
& +(1-w) \exp \left[-\left(\sigma_{\mathrm{BG}} t\right)^{2} / 2\right] \cos \left(\gamma_{\mu} B_{0} t\right),
\end{aligned}
$$

where

$$
P_{\mathrm{BCS}}(t)=\int_{0}^{\infty} n(z) \cos \left[\gamma_{\mu} B(z) t\right] d z,
$$

with

$$
B(z)=\left\{\begin{array}{l}
B_{0} \quad \text { when } z<z_{0}, \\
B_{\mathrm{BCS}}\left(z-z_{0}\right) \quad \text { when } z \geqslant z_{0},
\end{array}\right.
$$

and $z_{0}$ being the thickness of a "dead layer," an adjustable parameter that accounts for imperfections of the sample surface, such as an oxide layer and roughness, and for uncertainties of the Monte Carlo code TRIM.SP used for the calculation of the muon distribution $n(z)$. In this model the fitting parameter is not the field $B$, but the parameters of the chosen model for the field profile. In our case these parameters are $\lambda_{\mathrm{L}}$ and $z_{0}$.

\section{PNR}

PNR is an optical technique relying on the interaction of polarized neutrons with the magnetized sample interior. By measuring the intensity of the reflected neutron beam as a function of the incident neutron spin state and its wave vector it is possible to determine the magnetic field profile in a sample. At grazing angles of incidence the interaction can be described by a one-dimensional optical potential composed of a nuclear (neutron-nucleus interaction) and a magnetic contribution. The 

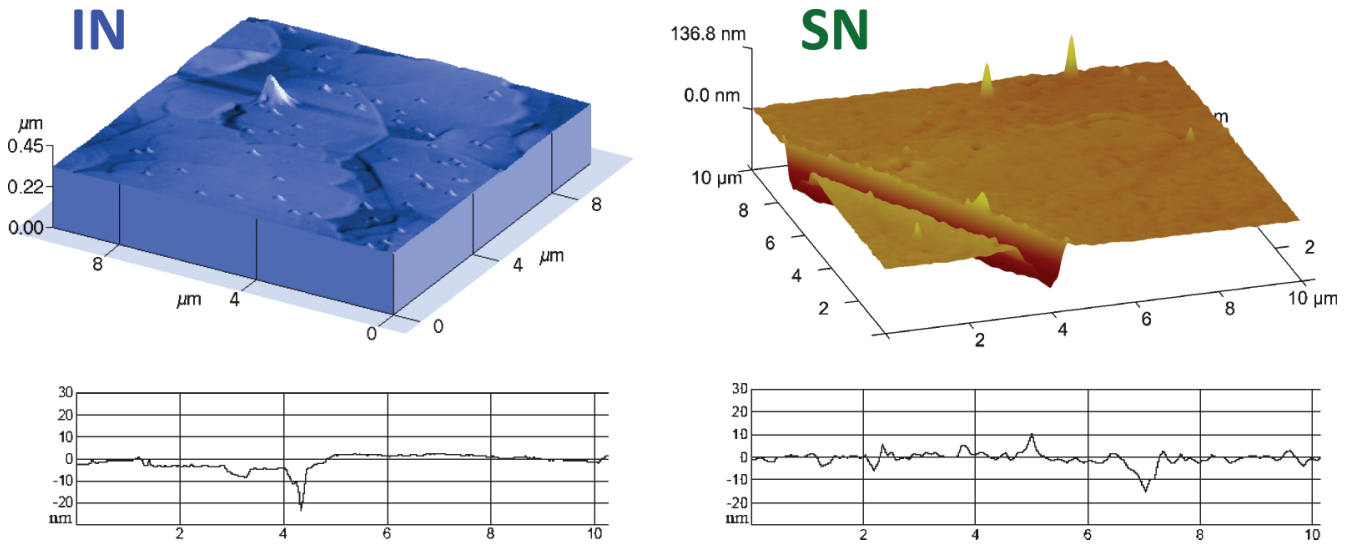

FIG. 5. (Color online) Typical AFM images and scans of the samples.

latter arises from the interaction of the neutron magnetic moment $\vec{\mu}$ and the magnetic field $\vec{B}$ inside the sample and is equal to $-\vec{\mu} \cdot \vec{B}$. Therefore, the reflectivity $R^{+}$of neutrons with spin parallel to $\vec{B}$ differs from the reflectivity $R^{-}$(the neutron spin is antiparallel to $\vec{B}$ ), and the difference $R^{+}-R^{-}$ depends on $B$.

Upon tuning the nonmagnetic parameters of the sample (effective surface roughness $\epsilon$, oxide layer, and sample thickness) and the neutron beam resolution on the reflectivity curve measured in the normal state, the spin asymmetry $s \equiv$ $\left(R^{+}-R^{-}\right) /\left(R^{+}+R^{-}\right)$is solely determined by the magnetic field profile $B(z)$. The effective roughness $\epsilon$ is a parameter of the model used to simulate the reflectivity in the normal state; it is related to but not the same as the physical roughness, such as, e.g., the root-mean-square roughness (rms) $\sigma$. In particular, $\epsilon \approx \sigma$ in case of uniform, short-wavelength roughness of nanometer scale as in high-quality polished surfaces, like in our Sn sample; but these quantities are only weakly related for the terrace-like surface structures, like in our In samples.

PNR operates with a polarized neutron beam incident under grazing angle $\theta$ near the critical angle $\theta_{c}$ of total external reflection. Therefore systematic instrumental errors in the
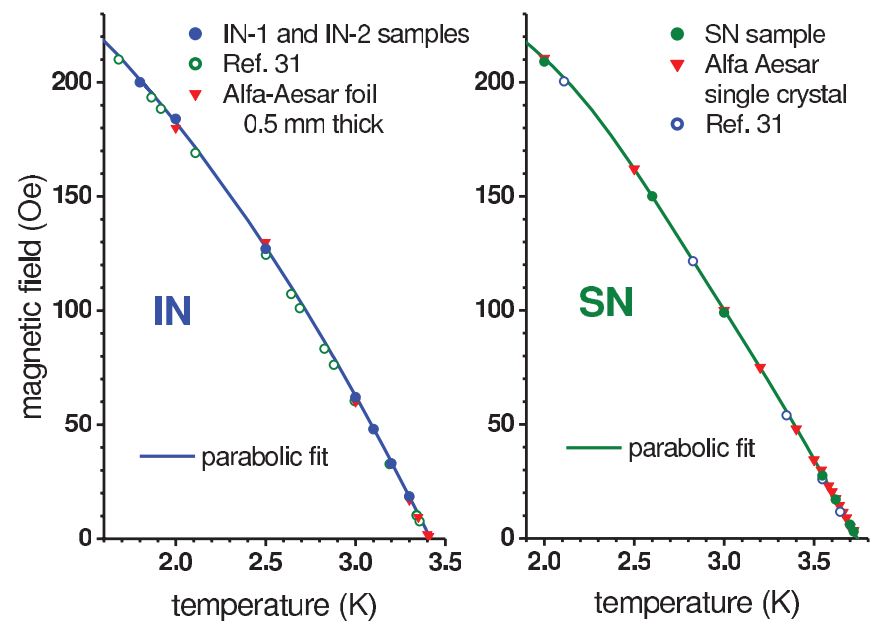

FIG. 6. (Color online) The phase diagrams of the indium and tin samples. angle of incidence and reflectivity are easily detected and corrected using Snell's law for $\theta_{c}$ and unity of the reflectivity for $\theta<\theta_{c}$, respectively. However, to obtain high-quality PNR data the sample surface should be large $\left(>3 \mathrm{~cm}^{2}\right)$, flat, and mirror-like smooth. Another challenge is associated with the necessity to use a highly collimated beam, which leads to a decrease of the neutron flux and therefore to a long exposure time (several days) needed to collect data sets with appropriate statistics. More technical details about neutron reflectometry can be found in Ref. 28; details about using PNR for measurements of $B(z)$ in superconductors are available in Ref. 10.

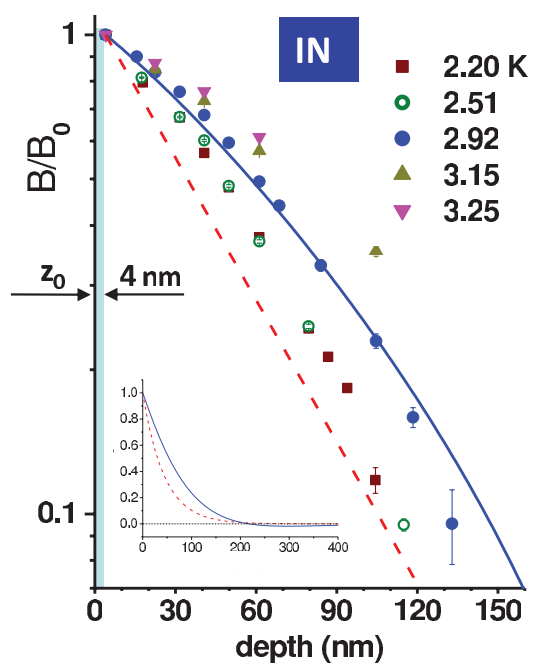

FIG. 7. (Color online) Reduced field $B / B_{0}$ in indium obtained from the LE- $\mu$ SR measurements using the Gaussian model. The depth is the average stopping distance of the implanted muons calculated from the Monte Carlo TRIM.SP code. The solid line is the field profile calculated from the nonlocal theory at $T=2.92 \mathrm{~K}$ with $\xi_{0}=380 \mathrm{~nm}$, $\lambda_{\mathrm{L}}(0)=30 \mathrm{~nm}$, and $z_{0}=4 \mathrm{~nm}$, inferred from the $\chi^{2}$ and the timedomain analysis. The dashed line represents $B(z) / B_{0}$ calculated from the local theory at the same temperature with the same values of $\lambda_{\mathrm{L}}(0)$ and $z_{0}$. The inset presents the same field profiles on a linear scale. 


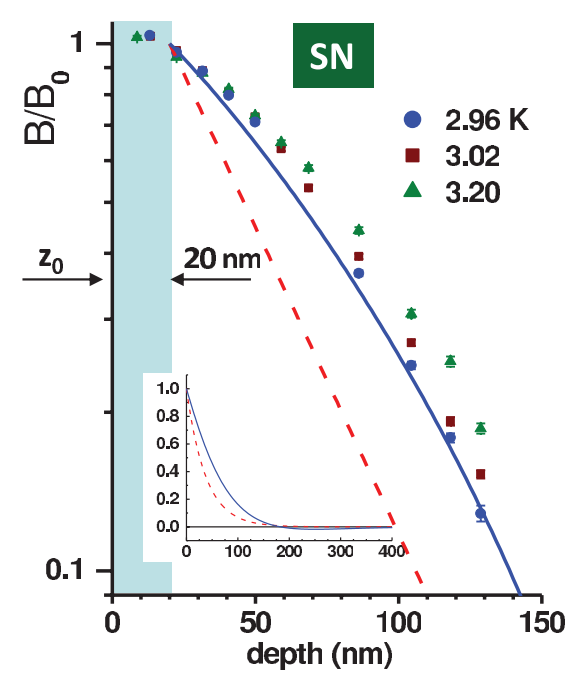

FIG. 8. (Color online) Reduced field $B / B_{0}$ in tin obtained from the LE- $\mu$ SR measurements using the Gaussian model. The depth is the average stopping distance of the implanted muons calculated from the Monte Carlo TRIM.SP code. The solid line represents the field profile calculated from the nonlocal theory at $T=2.96 \mathrm{~K}$ with $\xi_{0}=310 \mathrm{~nm}, \lambda_{\mathrm{L}}(0)=36 \mathrm{~nm}$, and $z_{0}=20 \mathrm{~nm}$, inferred from the $\chi^{2}$ and the time-domain analysis. The dashed line represents $B(z) / B_{0}$ calculated from the local theory at the same temperature with the same values of $\lambda_{L}(0)$ and $z_{0}$. The inset presents the same field profiles on a linear scale.

\section{SAMPLES}

The indium samples were films deposited on an oxidized silicon wafer (IN-1) and on a sapphire crystal (IN-2) held at room temperature via thermal evaporation of indium shots (Alfa-Aesar, $99.9999 \%$ purity) at a base pressure $\lesssim 5 \times$ $10^{-9}$ mbar. The IN-1 sample was used for PNR measurements and for LE- $\mu$ SR measurements down to $2.9 \mathrm{~K}$. The IN-2 sample was used for LE- $\mu$ SR measurements at lower temperatures. The tin sample was a polished single crystal (Surface Preparation Laboratory, The Netherlands); the same sample was used in both LE- $\mu$ SR and PNR experiments. Parameters of the samples are listed in Table I.

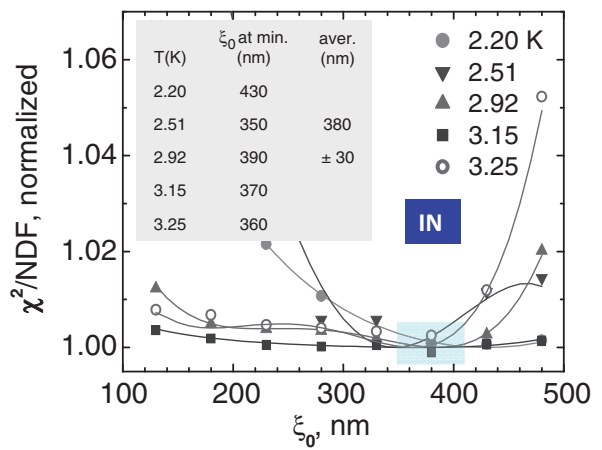

FIG. 9. (Color online) In samples: $\chi^{2} / \mathrm{NDF}$ versus $\xi_{0}$ and the corresponding polynomial fittings normalized to the minimum value for each temperature. The shaded area indicates the range of the best estimates for $\xi_{0}$. The inset table gives positions of the minima and their average with the standard deviation.

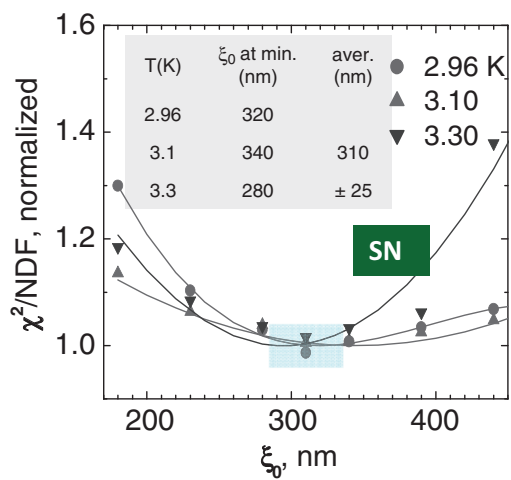

FIG. 10. (Color online) Sn sample: $\chi^{2} / \mathrm{NDF}$ versus $\xi_{0}$ and the corresponding polynomial fittings normalized to the minimum value for each temperature. The shaded area indicates the range of the best estimates for $\xi_{0}$. The inset table gives positions of the minima and their average with the standard deviation.

Magnetization data measured with a SQUID magnetometer on IN-1 and SN samples are shown in Fig. 3. The samples exhibit a clear-cut first-order phase transition and deep supercooling of the normal state under decreasing field, hence testifying to the high purity of the samples. The electrical resistance was measured with a low-current ac bridge. The resistance data obtained with the IN-2 sample are shown in Fig. 4; the very high purity of this sample is also evident from the fact that the resistance does not reach a temperatureindependent residual value at temperatures down to $T_{c} \cdot{ }^{29}$ The elastic mean free path $\ell$ was calculated from the measured residual resistivity ratio (RRR); the values of the product $\ell \rho$ ( $\rho$ is the resistivity) at room temperature necessary for that were taken from Ref. 30.

Typical AFM images and scans of indium and tin samples are shown in Fig. 5. The surface of the In films consists of nearly atomically flat terraces (the root-mean-square

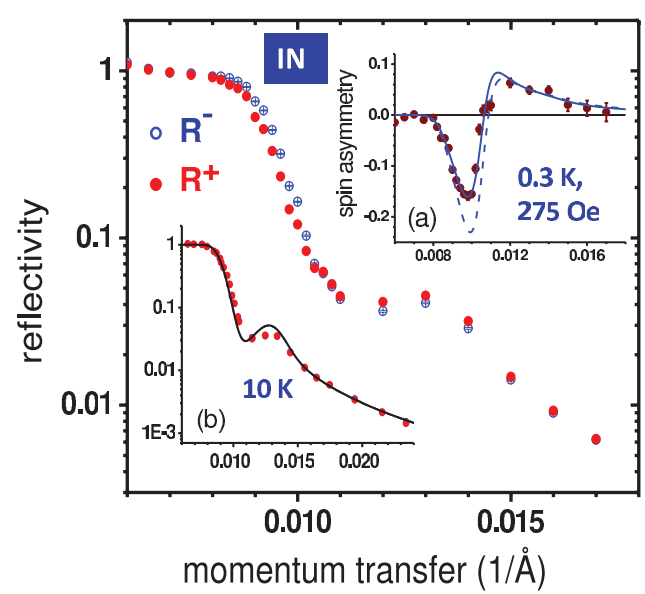

FIG. 11. (Color online) Reflectivity of neutrons polarized parallel $\left(R^{+}\right)$and antiparallel $\left(R^{-}\right)$to the magnetic field in the Meissner state in In at $T=0.3 \mathrm{~K}$ and $B_{0}=275 \mathrm{Oe}$. Inset (a): Measured spin asymmetry (points) and its simulation with $B(z)$ obtained from the local (dashed curve) and nonlocal (solid curve) theories with $\lambda_{\mathrm{L}}=$ $28 \mathrm{~nm}$ and $\xi_{0}=380 \mathrm{~nm}$. Inset (b): Measured (points) and simulated (curve) reflectivity in the normal state. 


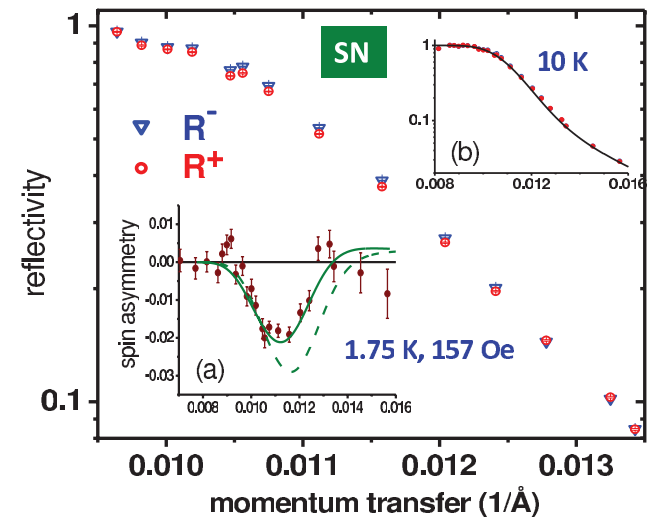

FIG. 12. (Color online) Reflectivity of neutrons polarized parallel $\left(R^{+}\right)$and antiparallel $\left(R^{-}\right)$to the magnetic field in the Meissner state in Sn at $T=1.75 \mathrm{~K}$ and $B_{0}=157 \mathrm{Oe}$. Inset (a): Measured spin asymmetry (points) and its simulation with $B(z)$ obtained from the local (dashed curve) and nonlocal (solid curve) theories with $\lambda_{\mathrm{L}}=37.5 \mathrm{~nm}$ and $\xi_{0}=310 \mathrm{~nm}$. Inset (b): Measured (points) and simulated (curve) reflectivity in the normal state.

roughness of the terrace areas is $\lesssim 2 \AA$ ) with a typical size of about $5 \mu \mathrm{m}$ with voids in between. This size is much larger than $\xi_{0}$ while the total area of the voids does not exceed $3 \%$ of the sample surface. Therefore the terrace surface structure should not affect the electrodynamic properties of the films. The surface of the polished Sn sample has a short-wavelength roughness well characterized by the rms roughness.

The phase diagrams of the samples used in this work along with other high-purity Alfa-Aesar In and Sn samples are shown in Fig. 6. The measured data on the thermodynamic critical field $H_{c}(T)$ and $T_{c}$ perfectly agree with values reported in the literature. ${ }^{31,32}$ For all samples the elastic mean free path $\ell>$ $10 \mu \mathrm{m} \gg \xi_{0}$; therefore the samples are type-I superconductors in the pure limit. ${ }^{2}$

\section{RESULTS AND DISCUSSION}

The $L E$ - $\mu S R$ experiments were performed at the $\mu \mathrm{E} 4$ beamline of the Swiss Muon Source at the Paul Scherrer Institute. ${ }^{33}$ The $B(z)$ points obtained using the Gaussian model for the depolarization of the precessing muons along with the $B(z)$

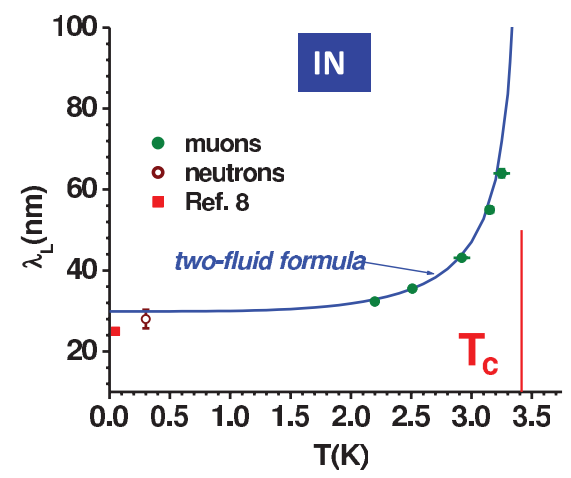

FIG. 13. (Color online) Temperature dependence of the London penetration depth in indium determined from the LE- $\mu$ SR (muons) and PNR (neutrons) measurements.

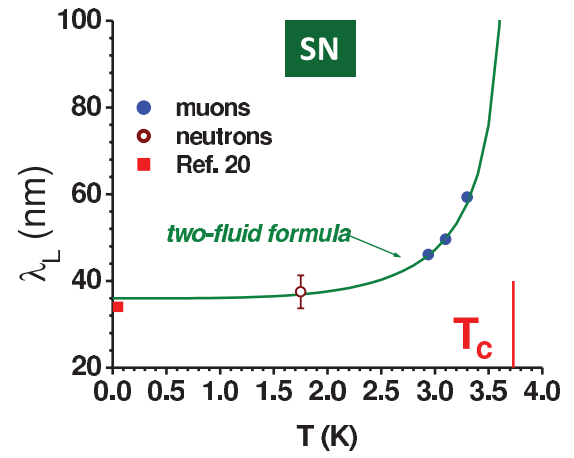

FIG. 14. (Color online) The London penetration depth in tin determined from the LE- $\mu$ SR (muons) and PNR (neutrons) measurements.

curve calculated from the local and nonlocal theories for In and Sn are presented in Figs. 7 and 8, respectively. The sample temperature was determined in situ based on the $H_{c}(T)$ phase diagram obtained from magnetization measurements (Fig. 6). As can be seen in Figs. 7 and 8, the "Gaussian" $B$ points exhibit a pronounced nonexponential depth dependence, consistent with the nonlocal effect. Discrepancies between the points and the "nonlocal" theoretical curves are mainly caused by incomplete adequacy of the Gaussian model. On the other hand, the qualitative consistency of the "Gaussian" points with the nonlocal theory justifies the application of the time-domain model, directly assuming the "nonlocal" shape of $B(z)$ with $\lambda_{\mathrm{L}}$ as an adjustable parameter.

In nonlocal superconductors the effective penetration depth is $\lambda_{\text {eff }}^{3} \approx \lambda_{\mathrm{L}}^{2} \xi_{0}^{2}$. Therefore either $\lambda_{\mathrm{L}}$ or $\xi_{0}$ has to be determined using an additional criterion. Since $\lambda_{\text {eff }}$ is mainly sensitive to $\lambda_{\mathrm{L}}$ and $\xi_{0}$ depends weakly on temperature, this parameter is $\xi_{0}$. Its optimal value was found from a $\chi^{2}$ analysis of the global fits (simultaneous fitting of all implantation energy data sets at each temperature). The fits were performed with different $\xi_{0}$ chosen around a theoretically estimated value of $377 \mathrm{~nm}$ for $\mathrm{In}^{8}$ and of $230 \mathrm{~nm}$ for $\mathrm{Sn}^{20}$ The graphs for $\chi^{2} / \mathrm{NDF}$ (NDF stands for number of degrees of freedom) versus $\xi_{0}$ are presented in Fig. 9 for In and in Fig. 10 for Sn. Values of $\xi_{0}$ at the minima and the best estimate for $\xi_{0}$ are given in the insets. The best estimate for the Pippard coherence length is $380 \pm 30 \mathrm{~nm}(\mathrm{In})$ and $310 \pm 25 \mathrm{~nm}(\mathrm{Sn})$. The values of $\lambda_{\mathrm{L}}$ obtained from the global fits with these values of $\xi_{0}$ are presented in summary graphs in Figs. 13 and 14.

The PNR measurements were performed on the D3 reflectometer at the NRU reactor in Chalk River. ${ }^{28}$ The experimental data and simulations for the reflectivity of neutrons polarized

TABLE II. Values of London penetration depth $\lambda_{\mathrm{L}}$, of Pippard coherence length $\xi_{0}$, and of the renormalization factor $Z$ in In and Sn obtained in this work. $Z$ in Ref. 1 was obtained from tunneling spectroscopy data; $Z$ in Ref. 8 was deduced from the McMillan's equation for $T_{c}$.

\begin{tabular}{lccrrr}
\hline \hline sample & $\lambda_{\mathrm{L}}(0)(\mathrm{nm})$ & $\xi_{0}(\mathrm{~nm})$ & \multicolumn{1}{c}{$Z$} & $Z^{1}$ & $Z^{8}$ \\
\hline In & $30 \pm 2$ & $380 \pm 30$ & $2 \pm 0.4$ & 1.81 & 1.55 \\
Sn & $36 \pm 4$ & $310 \pm 25$ & $1.8 \pm 0.5$ & 1.72 & \\
\hline \hline
\end{tabular}


parallel $\left(R^{+}\right)$and antiparallel $\left(R^{-}\right)$to the magnetic field and for the spin asymmetry $\left(R^{+}-R^{-}\right) /\left(R^{+}+R^{-}\right)$are presented in Figs. 11 and 12 for In and Sn, respectively. In agreement with our LE- $\mu$ SR results and previous PNR results, ${ }^{10}$ the neutron spin asymmetry [insets (a) in Figs. 11 and 12] simulated with $B(z)$ calculated from the nonlocal theory matches the experimental data significantly better than the simulation based on the London field profile. The "nonlocal" $B(z)$ was calculated with the values of $\xi_{0}$ obtained from the LE- $\mu$ SR data. The best match of the simulated spin asymmetry with the experimental data was achieved for $\lambda_{\mathrm{L}}(T=0.3 \mathrm{~K})=$ $28.0 \pm 2.5 \mathrm{~nm}(\mathrm{In})$ and for $\lambda_{\mathrm{L}}(T=1.75 \mathrm{~K})=37.5 \pm 3.8 \mathrm{~nm}$ (Sn).

Results for $\lambda_{\mathrm{L}}$ inferred from the LE- $\mu \mathrm{SR}$ and PNR measurements for In and $\mathrm{Sn}$ are shown in Figs. 13 and 14 , respectively. For both metals the values of $\lambda_{\mathrm{L}}(T)$ are consistent with each other and agree with the two-fluid formula $\lambda^{2}(T)=\lambda^{2}(0) /\left[1-\left(T / T_{c}\right)^{4}\right]^{2}$. The best estimate of $\lambda_{\mathrm{L}}(0)$ in In is $30 \pm 2 \mathrm{~nm}$; in $\mathrm{Sn}$ it is $36 \pm 4 \mathrm{~nm}$.

Having determined $\lambda_{\mathrm{L}}(0)$ and $\xi_{0}$ we calculated $Z$ from Eq. (4). The results are summarized in Table II, from which it can be concluded that for both In and $\mathrm{Sn}$ the values of $Z$ obtained in this work agree with the values obtained from electron tunneling data. This implies that our results quantitatively confirm the nonlocal electrodynamic effect and confirm the validity of Eq. (4).

\section{SUMMARY AND OUTLOOK}

High-resolution measurements of the Meissner state magnetic field profiles $B(z, T)$ were performed in two extreme type-I superconductors, indium and tin, combining LE- $\mu$ SR and PNR measurements. The $B(z)$ profiles quantitatively confirm the Pippard/BCS nonlocal electrodynamic effect predicted by Pippard six decades ago. The $B(z)$ data were used to determine the Pippard coherence length $\xi_{0}$, the renormalization factor $Z$ for the electron-phonon mass enhancement of the Cooper pairs, and the London penetration depth $\lambda_{\mathrm{L}}(T)$ in In and Sn. An equation (4) linking $\xi_{0}, \lambda_{\mathrm{L}}(0)$, and $Z$ is introduced and experimentally verified. As demonstrated, in nonlocal superconductors this expression allows one to infer the renormalization factor $Z$ from the $B(z)$ data. In local superconductors, including unconventional materials, for which $\xi_{0}$ cannot be measured, Eq. (4) allows one to determine $\xi_{0}$ from measured $\lambda_{\mathrm{L}}(0)$ utilizing, e.g., LE- $\mu$ SR spectroscopy and $Z$ using, e.g., electron tunneling spectroscopy.

Finally it is worth noting that in type-II superconductors the size of the Cooper pairs can, in principle, be deduced from $\mu$ SR spectra measured in the mixed state. However a specific procedure for that still needs to be developed. ${ }^{34}$ Equation (4) can be used to verify such a procedure.

\section{ACKNOWLEDGMENTS}

This work was supported by the National Science Foundation under Grant No. DMR 0904157; by the Research Foundation - Flanders (FWO-Vlaanderen); and by the European Commission under the 7th Framework Programme through the "Research Infrastructures" action of the "Capacities" Programme, NMI3-II Grant No. 283883. The PNR results presented herein are made possible by a reflectometer jointly funded by the Canada Foundation for Innovation (CFI), Ontario Innovation Trust (OIT), Ontario Research Fund (ORF), and the National Research Council Canada (NRC). J.O.I. acknowledges the support by KU Leuven Grant No. OT/11/063. *vladimir.kozhevnikov@tulsacc.edu

†Present address: KTH Royal Institute of Technology, ICT Materials Physics, Electrum 229, 16440 Kista, Sweden.

${ }^{1}$ J. P. Carbotte, Rev. Mod. Phys. 62, 1027 (1990).

${ }^{2}$ M. Tinkham, Introduction to Superconductivity (McGraw-Hill, New York, 1996).

${ }^{3}$ A. Suter, E. Morenzoni, N. Garifianov, R. Khasanov, E. Kirk, H. Luetkens, T. Prokscha, and M. Horisberger, Phys. Rev. B 72, 024506 (2005).

${ }^{4}$ A. B. Pippard, Proc. R. Soc. London A 216, 547 (1953).

${ }^{5}$ I. Kosztin and A. J. Leggett, Phys. Rev. Lett. 79, 135 (1997).

${ }^{6}$ V. L. Ginzburg and L. D. Landau, Zh. Eksp. Teor. Fiz. 20, 1064 (1950).

${ }^{7}$ J. Halbritter, Z. Physik 243, 201 (1971).

${ }^{8}$ K. S. Wood and D. Van Vechten, Nucl. Instrum. Methods Phys. Res. A 314, 86 (1992).

${ }^{9}$ K. E. Drangeid and R. Sommerhalder, Phys. Rev. Lett. 8, 467 (1962).

${ }^{10}$ V. F. Kozhevnikov, C. V. Giuraniuc, M. J. Van Bael, K. M. Temst, C. Van Haesendonck, T. M. Mishonov, T. Charlton, R. M. Dalgliesh, Yu. N. Khaidukov, Yu. V. Nikitenko, V. L. Aksenov, V. N. Gladilin, V. M. Fomin, J. T. Devreese, and J. O. Indekeu, Phys. Rev. B 78, 012502 (2008).
${ }^{11}$ K. E. Drangeid, R. Sommerhalder, H. Muller, and H. Seitz, IBM J. Res. Dev. 8, 13 (1964).

${ }^{12}$ R. E. Doezema, J. N. Huffaker, S. Whitmore, J. Slinkman, and W. E. Lawrence, Phys. Rev. Lett. 53, 714 (1984).

${ }^{13}$ R. E. Doezema, S. C. Whitmore, and J. N. Huffaker, Phys. Rev. B 34, 4614 (1986).

${ }^{14}$ G. P. Felcher, R. O. Hilleke, R. K. Crawford, J. Haumann, R. Kleb, and G. Ostrowski, Rev. Sci. Instrum. 58, 609 (1987).

${ }^{15}$ S. J. Blundell and J. A. C. Bland, Phys. Rev. B 46, 3391 (1992).

${ }^{16}$ E. Morenzoni, F. Kottmann, D. Maden, B. Matthias, M. Meyberg, T. Prokscha, T. Wutzke, and U. Zimmermann, Phys. Rev. Lett. 72, 2793 (1994).

${ }^{17}$ E. Morenzoni, T. Prokscha, A. Suter, H. Luetkens, and R. Khasanov, J. Phys.: Condens. Matter 16, S4583 (2004).

${ }^{18}$ A. Suter, E. Morenzoni, R. Khasanov, H. Luetkens, T. Prokscha, and N. Garifianov, Phys. Rev. Lett. 92, 087001 (2004).

${ }^{19}$ T. E. Faber and A. B. Pippard, Proc. R. Soc. London A 231, 336 (1955).

${ }^{20}$ R. Mezervey and B. B. Schwartz, in Superconductivity, Vol. 1, edited by R. D. Park (Marcel Dekker, Inc., New York, 1969).

${ }^{21}$ N. W. Ashcroft and N. D. Mermin, Solid State Physics (Holt, Rinehart, and Winston, 1976).

${ }^{22}$ F. Scheck, Phys. Rep. 44, 187 (1978). 
${ }^{23}$ D. R. Harshman, A. P. Mills, Jr., J. L. Beveridge, K. R. Kendall, G. D. Morris, M. Senba, J. B. Warren, A. S. Rupaal, and J. H. Turner, Phys. Rev. B 36, 8850 (1987).

${ }^{24} \mathrm{~W}$. Eckstein, Computer Simulation of Ion-Solid Interactions (Springer Verlag, 1991).

${ }^{25}$ E. Morenzoni, H. Glückler, T. Prokscha, R. Khasanov, H. Luetkens, M. Birke, E. M. Forgan, Ch. Niedermayer, and M. Pleines, Nucl. Instrum. Methods Phys. Res. B 192, 254 (2002).

${ }^{26}$ R. F. Kiefl, M. D. Hossain, B. M. Wojek, S. R. Dunsiger, G. D. Morris, T. Prokscha, Z. Salman, J. Baglo, D. A. Bonn, R. Liang, W. N. Hardy, A. Suter, and E. Morenzoni, Phys. Rev. B 81, 180502 (2010).

${ }^{27}$ O. Ofer, J. C. Baglo, M. D. Hossain, R. F. Kiefl, W. N. Hardy, A. Thaler, H. Kim, M. A. Tanatar, P. C. Canfield, R. Prozorov,
G. M. Luke, E. Morenzoni, H. Saadaoui, A. Suter, T. Prokscha, B. M. Wojek, and Z. Salman, Phys. Rev. B 85, 060506(R) (2012).

${ }^{28} \mathrm{H}$. Fritzsche, in Characterization of Materials, edited by E. N. Kaufmann (John Wiley and Sons, 2012).

${ }^{29}$ H. Kamerlingh Onnes, Nobel lecture, 1913.

${ }^{30}$ G. K. Chang and B. Serin, Phys. Rev. 145, 274 (1966).

${ }^{31}$ D. K. Finnemore and D. E. Mapother, Phys. Rev. 140, A507 (1965).

${ }^{32}$ Handbook of Physical Quantities, edited by I. S. Grigoriev and E. Z. Meilikhov (CRC, New York, 1997).

${ }^{33}$ T. Prokscha, E. Morenzoni, K. Deiters, F. Foroughi, D. George, R. Kobler, A. Suter, and V. Vrankovic, Nucl. Instrum. Methods Phys. Res. A 595, 317 (2008).

${ }^{34}$ J. E. Sonier, Rep. Prog. Phys. 70, 1717 (2007). 